indices of functional decline in determining transplantation outcome in patients with cirrhosis.

Methods Twenty-eight consecutive patients (mean [range] age 52 [29-66] yr; 75\% men; four (14.3\%) alcohol-related; mean MELD 13.2 [7-30]) transplanted for end stage liver disease/ HCC were included. All were assessed pre-transplantation, as follows: disease severity: MELD and Child Pugh (CP); nutritional status: The Royal Free Hospital-Nutritional Prioritising Tool; HRQOL: Chronic Liver Disease Questionnaire and Euro Quol-5 Dimension Tool; mental health: Beck Anxiety and Depression Indices; disability: Activities (ADL) and Independent Activities of Daily Living (IADL); and frailty: Clinical Frailty Scale, Short Physical Performance Battery and Fried Frailty Criteria plus two composite instruments, the Bristol Prognostic Index and Karnofsky Age MELD Model. Variables associated with the primary outcome (death/retransplantation) were identified using Cox regression analysis. Variables associated with secondary outcomes, including the total units of blood transfused and the length of hospital stay, were identified using linear regression analysis.

Results Patients were followed for a mean of 143 [3-326] days; two (7.1\%) died and four (14.3\%) were retransplanted. IADL was the only tool significantly associated with mortality in this cohort. Each unit increase in the IADL (decreasing frailty) was associated with a $45 \%$ decrease in mortality after adjustment for MELD (Hazard Ratio (HR) 0.55, 95\% CI, 0.33-0.92). The total mean LOHS was 28 [7-112] days. The CP score was significantly associated with LOHS $(\mathrm{F}(1,25)$ $=6.01, \mathrm{p}=0.02, \mathrm{R}^{2}=0.19$ ); each unit increase in $\mathrm{CP}$ was associated with an increase in LOHS of 6.5 days. The mean units of blood transfused was 46 [3-178]; The amount transfused increased by $11.33(p=0.03)$ and $4.2(p=0.01)$ units for each unit increase in CP and MELD scores respectively.

Conclusions Disease severity and functional decline, characterised by the IADL score are significantly associated with short to medium term transplant outcomes in this cohort. Longerterm follow is required to validate these Results.

\section{PTH-100 HEPATITIS C IN AN INNER CITY HUB: REAL LIFE RESULTS}

Juhee Pahuja*, Susanne Johansen, Sam Douthwaite, Ranjababu Kulasegaram, Philip Berry, Terence Wong. Guy's and St Thomas' Healthcare NHS Trust, London, UK

\subsection{6/gutjnl-2018-BSGAbstracts.256}

Introduction Around 200000 people in the UK are infected with Hepatitis C (HCV). Recent advances in direct-acting antiviral (DAA) agents have revolutionised treatment of HCV with all oral regimens showing high cure rates. Registry studies of DAAs have reported sustained virologic response (SVR) rates of $>95 \%$. This study examines real life SVR rates outside of clinical studies in an inner city area.

Methods 250 eligible patients with chronic HCV were treated with DAAs from $10 / 09 / 2015$ to $27 / 07 / 2017$. After assigning to them to specific DAA combination therapy dependent on their genotype (GT), the hepatitis C RNA was measured 12 weeks after the cessation of therapy, with a SVR defined as an undetectable viral load (Roche amplicor, lower limit of detection $15 \mathrm{iu} / \mathrm{ml})$. We analysed the SVR12 according to genotype, treatment naïve versus previously treated patients, and specific DAA treatment combinations.
The mean age was 52 year (27 yr-84yr). 74\% (185/250) were male. 188/250 (75\%) patients were Caucasian. 5\%, 68\% and $27 \%$ of the patients had a Fibroscan liver stiffness measurement (LSM) of $<9.5 \mathrm{kPa}, 9.5 \mathrm{kPa}-11.5 \mathrm{kPa}$ and $>11.5 \mathrm{kPa}$, respectively (mean LSM $10 \mathrm{kPa}$ ). 91/250 (39\%) patients were HIV co-infected. There were 186 (74\%), 8 (3\%), 23 (9\%), 32 $(13 \%)$ and $1(0.4 \%)$ patients with HCV GT1, GT2, GT3, GT4 and GT5, respectively. The mean HCV RNA viral load was $\log 5.81 \mathrm{IU} / \mathrm{mL}$ (range: $\log 2.16$ to $\log 7.42 \mathrm{IU} / \mathrm{mL}$ ).

Results 94\% (234/250 patients) of the $250 \mathrm{HCV}$ infected patients achieved SVR12.

Across the specific treatment combinations+/-Ribavirin, $100 \%$ of 14 patients on Sofosbuvir and Velpatasvir, 96\% of 79 patients on Sofosbuvir and Ledipasvir, 91\% of 11 patients on Sofosbuvir and Daclatasvir, $86 \%$ of 7 patients on Sofosbuvir, 95\% of 37 patients on Elbasvir and Grazoprevir, 93\% of 85 patients Ombitasvir, Paritaprevir, Ritonavir and Dasabuvir, $88 \%$ of 17 patients on Ombitasvir, Paritaprevir and Ritonavir achieved SVR12.

94\% of 175 GT1 patients, $88 \%$ of 7 GT2 patients, 96\% of 22 GT3 patients, 91\% of 29 GT4 patients and the 1 GT5 patient achieved SVR12. Overall, 92 patients had previous treatment with $92 \%$ achieving SVR12. Out of the 158 treatment naïve patients, 95\% achieved SVR12.

There were 16 treatment failures overall; 1 due to poor treatment compliance and 6 responding, with relapse. 5 were lost in follow up with 1 patient achieving SVR4 and 1 with an end-dose response but subsequently both were lost in follow up. 1 patient discontinued due to acute cholecystitis and 2 patients died during the treatment period. 1 patient died after achieving SVR12.

Conclusions Our Results indicate DAA therapy is highly effective with real life SVR rates comparable to the registry studies, paving the way for HCV eradication in the UK.

\section{PTH-101 ALPPS: TECHNIQUE TO MINIMISE SMALL FOR SIZE SYNDROME AFTER MAJOR HEPATECTOMY FOR NEUROENDOCRINE TUMOUR METASTASES}

${ }^{1}$ Michail Pizanias*, ${ }^{2}$ Michail Papamichail, ${ }^{1}$ Elissaios Kontis, ${ }^{1}$ Evangelos Prassas, ${ }^{1}$ John Ramage, ${ }^{1}$ Parthi Srinivasan, ${ }^{1}$ Rajaventhan Srirajaskanthan, ${ }^{1}$ Andreas Prachalias. ${ }^{1}$ King's College Hospital, London, UK; ${ }^{2}$ Department of Organ Transplantation and HepatoPancreato- Biliary Surgery, Lahey Hospital and Medical Center, Boston, USA

10.1136/gutjnl-2018-BSGAbstracts.257

Introduction Hepatic resection has emerged as an effective treatment for secondary liver neuroendocrine tumours. 'Associated liver partition and portal vein ligation for staged hepatectomy' (ALPPS) allows resection of liver tumours in two steps. We present our experience in ALPPS procedure as a Method which can minimise small for size syndrome, and provide an oncological benefit to borderline resectable neuroendocrine tumours within acceptable safety profile.

Methods 4 patients (male: female: 1:1) underwent ALPPS procedure for clearance of the metastatic liver disease. Liver segments I, IV-VIII were resected for each patient. Two of the patients had bi-lobar disease. Clearance to future liver remnant (FLR) was achieved with non-anatomical liver resection in one case and with irreversible electroporation to the other as the lesion was adjacent to the left hepatic vein during the 1 st stage of the ALPPS procedure. Two patients underwent ALPPS 
as a salvage procedure after failed portal vein embolization and portal vein ligation respectively.

Results Median increase of FLR volume was 139.25\% (range 40.00\%-157.78\%). Median hospital stay was 28.5 days (range 23-36). Histology report revealed two complete (R0) and two incomplete (R1) resections. Background liver histology revealed steatosis on two occasions and fibrosis on another. 90 day mortality was zero. Two patients developed grade II complications as per Clavien-Dindo classification, one grade IIIa and one IIIb. One patient is disease free after 36 months,. One patient died two years later from viral infection, and two had recurrence in liver and lymph nodes and were treated with microwave ablation and lymphadenectomy respectively.

Conclusions ALPPS procedure allows performing major liver resections for high volume neuroendocrine metastases while minimising the risk of post-operative liver failure.

\section{PTH-102 CIRRHOTIC PATIENTS WITH VITAMIN D DEFICIENCY FAIL TO RESPOND TO ORAL REPLACEMENT THERAPY}

Freya Rhodes*, Olivia Greenham, Emma Hunt, Tina Shah, David Patch, Jennifer Ryan, Rachel Westbrook. Royal Free London NHS Foundation Trust, London, UK

\subsection{6/gutjnl-2018-BSGAbstracts.258}

Introduction Vitamin D deficiency and reduced BMD are highly prevalent in patients with advanced chronic liver disease. For bisphosphonate treatment for osteoporosis to be effective vitamin D levels must be replete. Moreover, vitamin $\mathrm{D}$ deficiency has been associated with an increased risk of infections and increased rejection rates following liver transplantation. The optimal dose and route of vitamin D replacement in cirrhosis is unknown. BSG guidance currently recommends $800 \mathrm{IU} /$ day orally for all patients with cirrhosis/ cholestatic liver disease.

Methods Retrospective review of 218 cirrhotic patients undergoing evaluation for liver transplant between 2016 and 2017. Vitamin D 'severe deficiency' was defined as $<25 \mathrm{ng} / \mathrm{ml}$, 'deficiency' $25-50 \mathrm{ng} / \mathrm{ml}$ and normal $>50 \mathrm{ng} / \mathrm{ml}$. Response to oral vitamin D therapy was recorded.

Results Out of 218 patients, 128/218 (59\%) had low Vitamin D levels with 25\% $(\mathrm{n}=55) \quad$ 'severely deficient' and $33 \%$ $(\mathrm{n}=73)$ 'deficient'. Overall 33 patients with levels $<50 \mathrm{ng} / \mathrm{ml}$ $(52 \%)$, and 31 patients $(48 \%)$ with levels $>50 \mathrm{ng} / \mathrm{ml}$ received replacement therapy. $(p=0.86)$

Median daily dose of Vitamin D replacement was 2800 units/day (IQR 800-2800) in $<25 \mathrm{ng} / \mathrm{ml}$ group, 2860 units/ day (IQR 800-2800) in $<50 \mathrm{ng} / \mathrm{ml}$ group and 800 units/day (IQR 800-2000) in $>50 \mathrm{ng} / \mathrm{dl}$ group. No significant difference in dosing between these groups $(p=0.12)$.

Data on vitamin D levels pre and post 3 months of treatment with Vitamin D therapy were available in 58 patients. Patients received either 400IU/day $(n=6), 800-1600 \mathrm{IU} /$ day $(n=28)$ or $>1600 \mathrm{IU} /$ Day $(n=24)$. Median delta change in vitamins $D$ levels in the 3 groups were $-3 \mathrm{ng} / \mathrm{ml},-1 \mathrm{ng} / \mathrm{ml}$ and $12 \mathrm{ng} / \mathrm{ml}$ over the 3 month treatment period. An average daily dose of $>1600 \mathrm{IU} /$ day Resulted in a significantly greater increase in Vitamin D levels when compared to doses $<1600$ IU/day $(p=0.01)$, albeit still sub optimal with only a median increase of $12 \mathrm{ng} / \mathrm{ml}$.

When those patients with Vitamin D levels of $<50 \mathrm{ng} / \mathrm{ml}$ were reviewed in isolation $(n=29), 82 \%$ failed to augment vitamin $\mathrm{D}$ levels to within the normal range $>50 \mathrm{ng} / \mathrm{dl}$ and no significant difference was found between dosages of vitamin D administered.

Conclusion Vitamin D deficiency is prevalent, affecting over $50 \%$ of patients with advanced cirrhosis. Oral vitamin D replacement therapy is ineffective in cirrhotics at repleting stores over a 3 month period irrespective of dose given.

Future evaluation of efficacy of IM administration in this unique cohort of patients is urgently needed to evaluate if this allows normalisation of Vitamin D levels.

\section{PTH-103 EPIDEMIOLOGY OF VITAMIN D DEFICIENCY AND BONE MINERAL DENSITY IN PATIENTS WITH CHRONIC LIVER DISEASE}

Freya Rhodes*, Olivia Greenham, Emma Hunt, Orla Kearney, David Patch, Jennifer Ryan, Rachel Westbrook. Royal Free NHS Foundation Trust, London, UK

\subsection{6/gutjnl-2018-BSGAbstracts.259}

Introduction Patients with chronic liver disease have increased risk of bone disease (BD) with reported prevalence of osteoporosis (OP) between $12 \%-55 \%$ and a high prevalence of Vitamin D deficiency. The aetiology is poorly understood with a complex interplay between endocrine, metabolic, nutritional and physical abnormalities. We aim to evaluate the influence of epidemiological parameters on bone mineral density and vitamin D levels in cirrhotics.

Methods Retrospective study of cirrhotic patients from 20162017. Data were collected on aetiology of cirrhosis, severity (UKELD/MELD score), bone mineral density (BMD), vitamin $\mathrm{D}$, body mass index (BMI) and hand grip strength. OP was defined as per WHO classification and Vitamin D deficiency as a Vitamin D level $<50 \mathrm{nmol} / \mathrm{L}$ with severe deficiency $<25 \mathrm{nmol} / \mathrm{L}$.

Results 248 patients were included, 180 male, 58 female, median age 57 years (IQR 49-63) and median BMI of 27. Underlying aetiology was ALD $(n=78)$, Viral $(n=56), \mathrm{PBC} /$ PSC $(n=46)$, NAFLD $(n=23)$ and AIH $(n=18)$. Median UKELD and MELD scores overall were 53 (IQR 49-57) and 14 (IQR 10-19). At the time of evaluation 141 (56.8\%) patients were either osteoporotic $(n=52)$ or osteopenic $(\mathrm{n}=99)$. The prevalence of BD was significantly higher in cholestatic diseases $(71.7 \%$, mean $\mathrm{T}$ score $-1.86+1.22)$ and lower in NAFLD (37\%, mean T score $-0.45+/-1.50)$ when compared to other aetiologies (ANOVA $p=0.0005) .120$ $(55.4 \%)$ patients were vitamin D deficient with 51 (25\%) patients having severe deficiency. Mean vitamin D level was highest in cholestatic disease $(75.5 \mathrm{ng} / \mathrm{ml}+/ 59.6)$ when compared to other aetiologies (ANOVA $\mathrm{p}=0.003$ ).

Liver severity scores (UKELD/MELD respectively) did not correlate with the presence of $B D(p=0.32 / p=0.53)$ but patients with higher MELD scores had lower vitamin D levels $(p=0.04)$. Reduced BMI correlated with the presence of BD $(\mathrm{p}<0.01)$ but not vitamin D level. Increased Hand Grip Strength (HGS) was associated with higher vitamin D levels $(p=0.049)$ and higher lumbar $T$ scores $(p=0.014)$. Vitamin D levels did not correlate with BMD $(p=0.77)$.

Conclusion Bone disease and vitamin D deficiency are prevalent in patients with cirrhosis, with cholestatic aetiologies having the highest prevalence of OP and NAFLD the lowest. 